\title{
Cahn-Hilliard-type phase-field theory for species diffusion coupled with large elastic deformations: application to phase-separating Li-ion electrode materials
}

Anand, Lallit, anand@mit.edu, MIT

\begin{abstract}
We formulate a unified framework of balance laws and thermodynamically consistent constitutive equations which couple Cahn-Hilliard-type species diffusion with large elastic deformations of a body. The traditional Cahn-Hilliard theory, which is based on the species concentration and its spatial gradient leads to a partial differential equation for the concentration which involves fourth-order spatial derivatives in the concentration; this necessitates use of basis functions in finite-element solution procedures that are piecewise smooth and globally $\mathrm{C}_{1}$-continuous. In order to use standard $\mathrm{C}_{0}$-continuous finite-elements to implement our phase-field model, we use a split method to reduce the fourth-order equation into two second-order partial differential equations (PDES). Specifically, we introduce an additional variable - as in the micromorphic theories of continua - and formulate a theory which depends on actual concentration, the micromorphic concentration, and the gradient of the micromorphic concentration. These two PDES, when taken together with the PDE representing the balance of forces, represent the three governing pdes for chemomechanically coupled problems. These equations are amenable to finite-element solution methods which employ standard $\mathrm{C}_{0}$-continuous finite-element basis functions. We have numerically implemented our theory by writing a user-element subroutine for the widely used finite-element program Abaqus/Standard. We use this numerically implemented theory to first study the diffusion-only problem of spinodal decomposition in the absence of any mechanical deformation. Next, we use our fully-coupled theory and numerical-implementation to study the combined effects of diffusion and stress on the lithiation of a representative spheroidal-shaped particle of a phase-separating electrode material.
\end{abstract}

